\title{
PROJETO DE SAÚDE NO TERRITÓRIO COMO ESTRATÉGIA PARA PROMOÇÃO DA CULTURA DE PAZ
}

HEALTH PROJECT IN THE TERRITORY AS A STRATEGY FOR PROMOTING THE CULTURE OF PEACE

PROYECTO DE SALUd EN EL TERRITORIO COMO UNA ESTRATEgIA PARA PROMOCIÓN DE LA CULTURA DE LA PAZ

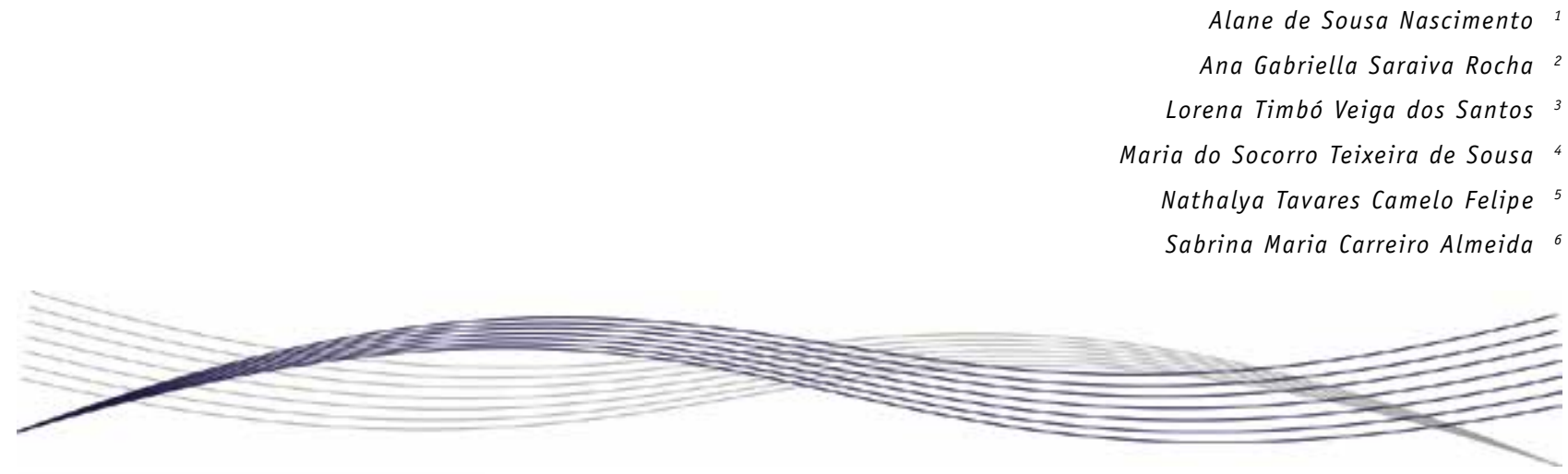

Palavras-chave: Participação Social; Estratégia Saúde da Família; Ação Intersetorial.

Keywords: Social Participation; Family Health Strategy; Intersectoral Action.

Palabras clave: Participación Social; Estrategia Salud de la Familia; Acción Intersectorial.

Submetido: 23/06/2018

Aprovado: $11 / 12 / 2018$

Autor(a) para Correspondência: Lorena Timbó Veiga dos Santos

R. Ressurreição, 720 Domingos Olímpio Sobral-CE

CEP:62022345 E-mail: lorenatveiga@gmail.com

\section{RESUMO}

Este artigo relata uma experiência vivenciada na construção de um Projeto de Saúde no Território (PST) no Complexo Sinhá Sabóia, em Sobral-CE. Trata-se de relato de experiência que descreve a utilização do PST como ferramenta tecnológica de apoio à atenção à saúde, com foco na organização de um Fórum Social da Juventude (FSJ) para discutir ações relacionadas à cultura de paz, com a participação de lideranças comunitárias, equipes dos Centros de Saúde da Família (CSF) "Sinhá Sabóia" e "Cohab II", escolas das redes municipal e estadual e os conselhos municipal e local de saúde. As ações contemplaram as 3 etapas do PST: a) preparação; b) planejamento/ implementação; e c) avaliação. A violência se mostrou um determinante social de saúde no território, o que fomentou estratégias de gestão e estabeleceu redes de cogestão e corresponsabilidade, produzindo espaços de reflexão sobre a situação dos adolescentes do bairro e potencializando ações como direito de cidadania, além de incentivar o protagonismo juvenil para uma cultura de paz. O PST estimulou a prática do planejamento e da avaliação em saúde, possibilitando a congregação dos diversos setores e do poder público e instigando o diálogo sobre questões inerentes aos modos $e$ às condições de vida da população.

\footnotetext{
1. Nutricionista. Especialista em Saúde da Família pela Escola de Formação em Saúde da Família Visconde de Sabóia (EFSFVS). Sobral (CE), Brasil. E-mail: alanny-18@hotmail.com

2. Assistente Social. Especialista em Saúde da Família pela EFSFVS. Aluna de Mestrado em Gestão Pública pela Universidade Federal do Piauí (UFPI). Teresina (PI), Brasil. E-mail: ana.gabriella2@hotmail.com 3. Enfermeira. Especialista em Saúde da Família pela EFSFVS. Residente em Urgência e Emergência na Santa Casa de Misericórdia de Sobral pelo Centro Universitário Inta (Uninta). Sobral (CE), Brasil. E-mail: lorenatveiga@ gmail.com

4. Enfermeira. Mestre em Saúde da Família pela Univ. Estadual Vale do Acaraú (UVA). Tutora da Residência Multiprofissional em Saúde da Família (RMSF) da EFSFVS. Sobral (CE), Brasil. E-mail: socorrinhoteixeira@ yahoo.com.br

5. Fonoaudióloga. Especialista em Saúde da Família pela EFSFVS. Residente em Neurologia e Neurocirurgia pela Escola de Saúde Pública do Ceará (ESP/CE). Fortaleza (CE), Brasil. E-mail: nathalyatcf@gmail.com 6. Farmacêutica. Especialista em Saúde da Família pela EFSFVS. Sobral (CE), Brasil. E-mail: sabrinalmeida82@ hotmail.com
} 


\section{ABSTRACT}

This article reports a lived experience in the construction of a health project in the territory (HPT) in the Sinhá Sabóia Complex, in Sobral, Ceará, Brazil. This is an experience report that describes the use of a HPT as a technological tool to support health care, focusing on the organization of a social youth forum (SYF) to discuss actions related to the culture of peace, relying on the participation of community leaders, teams from the family health care centers (FHCCS) 'Sinhá Sabóia' and 'Cohab II,' municipal and state schools, and municipal and local health councils. Actions included the 3 HPT stages: a) preparation; b) planning/implementation; and c) evaluation. Violence proved to be a social determinant of health in the territory, and this fostered management strategies and established co-management and co-responsibility networks, producing spaces for reflection on the situation of teenagers in the neighborhood and strengthening actions as a right of citizenship, as well as encouraging juvenile protagonism for a culture of peace. The HPT stimulated the practice of health planning and evaluation, enabling the congregation of the various sectors and public power and instigating dialogue on issues inherent to the population's living standards and conditions of life.

\section{RESUMEN}

Este artículo reporta una experiencia vivida en la construcción de un proyecto de salud en el territorio (PST) en el Complejo Sinhá Sabóia, en Sobral, Ceará, Brasil. Este es un informe de experiencia que describe el uso de un PST como una herramienta tecnológica de apoyo a la atención a la salud, con foco en la organización de un foro social de la juventud (FSJ) para discutir acciones relacionadas con la cultura de la paz, apoyándose en la participación de líderes comunitarios, equipos de los centros de salud de la familia (CSF) "Sinhá Sabóia" y "Cohab II", escuelas municipales y del estado y los consejos de salud municipales y locales. Las acciones incluyeron las 3 etapas del PST: a) preparación; b) planificación/implementación; y c) evaluación. La violencia demostró ser un determinante social de la salud en el territorio, lo que fomentó las estrategias de gestión y estableció redes de co-gestión y co-responsabilidad, generando espacios para la reflexión acerca de la situación de los adolescentes del barrio y fortaleciendo las acciones como un derecho de ciudadanía, así como fomentando el protagonismo juvenil para una cultura de la paz. El PST estimuló la práctica de planificación y evaluación de la salud, lo que permitió la congregación de los diversos sectores y del poder público e instigó el diálogo acerca de cuestiones inherentes a los modos y las condiciones de vida de la población.

\section{INTRODUÇÃO}

As ferramentas tecnológicas em saúde foram idealizadas como recursos de organização e ampliação do trabalho das equipes da Estratégia Saúde da Família (ESF). Dentre tais ferramentas se destaca o Projeto de Saúde no Território (PST), que busca investir na criação de espaços democráticos de participação social e na emancipação de sujeitos e comunidades ${ }^{1}$.

0 PST incentiva as ações voltadas à prevenção de doenças e à promoção da saúde, bem como à redução de fatores de vulnerabilidade de uma área de abrangência, fomentando a qualidade de vida da comunidade e o protagonismo dos indivíduos, por meio da cogestão entre a ESF, o Núcleo Ampliado de Saúde da Família e Atenção Básica (Nasf-AB), os outros serviços de saúde e as políticas sociais ${ }^{1}$.

Sob essa perspectiva, o PST se fundamenta em 3 pilares: a) promoção da saúde; b) participação social; e c) intersetorialidade. Mostra-se necessário um planejamento contínuo e coletivo para a construção do PST, com pactuação entre os atores envolvidos no processo para definir as responsabilidades de cada um. E os encontros devem ser periódicos, proporcionando espaço para o diálogo, pois a avaliação da implementação, da execução e dos resultados é imprescindível ${ }^{2}$.

Os atores envolvidos na ação devem tomar parte no planejamento, uma vez que têm contato diário com o trabalho e sabem exatamente o que é exequível ou não, por isso a figura do "planejador" não deve ser idealizada como a de um ator principal, mas sim como a de um facilitador do processo junto aos atores ${ }^{3}$. Também se considera que o planejamento constitui instrumento governamental com vistas a aprimorar a produção de políticas, a gestão de órgãos públicos e a práxis social 4 .

Ao assumir a dimensão como ação social, coletiva e institucional, o planejamento se torna crucial para a melhoria dos processos de trabalho e oportuno para explicitar objetivos e compromissos compartilhados 
em um arcabouço de justificativas políticas e institucionais ${ }^{5}$.

Em saúde, sabe-se que a avaliação é tão importante quanto o planejamento. Entende-se a avaliação como um processo contínuo e sistemático que proporciona espaço crítico e reflexivo acerca das práticas nos serviços de saúde ${ }^{6}$. Desse modo, o processo avaliativo envolve pluralismo metodológico para responder às necessidades em saúde dos diversos atores e é constituído por múltiplos elementos ${ }^{7}$.

Para a construção bem-sucedida do PST como ferramenta tecnológica em saúde, de grande relevância na atenção básica, faz-se necessário combinar as práticas de planejamento e avaliação.

Nesse contexto, compreende-se que o PST é um instrumento-chave para disparar processos de mudanças nas práticas em saúde, contribuindo para diversificar os serviços disponíveis, bem como criar espaços coletivos para discussão e análise de aspectos como a priorização das necessidades de saúde específicas, seus determinantes sociais, as estratégias e os objetivos para sua abordagem.

Este artigo relata uma experiência vivenciada na construção de um PST, por meio da realização de um Fórum Social da Juventude (FSJ) para discutir ações relacionadas à cultura de paz em Sobral-CE.

\section{METODOLOGIA}

Trata-se de relato de experiência que descreve a utilização do PST, ferramenta tecnológica de apoio à atenção à saúde, com foco na organização de um FSJ para discutir ações relacionadas à cultura de paz dentro da comunidade em Sobral.

Para a construção desse PST, recorreu-se à congregação de esforços e à articulação intersetorial, com a participação de lideranças comunitárias, equipes de saúde dos Centros de Saúde da Família (CSF) "Sinhá Sabóia" e "Cohab II", escolas das redes municipal e estadual e os conselhos municipal e local de saúde, todos reunidos como Conselho Local de Saúde, destacando-se o protagonismo da equipe de Residência Multiprofissional em Saúde da Família (RMSF) e sua tutoria nesse território.

0 desenvolvimento do PST contemplou as 3 etapas indicadas pelo Caderno de Atenção Básica do Ministério da Saúde ${ }^{1}$ a) preparação; b) planejamento/ implementação; e c) avaliação.

A primeira etapa, preparação, iniciou-se pela identificação de uma situação de vulnerabilidade social, seguida pela apresentação e consolidação de

\section{... PST é um \\ instrumento-chave para \\ disparar processos de \\ mudanças nas práticas \\ em saúde...}

um entendimento mais aprofundado da situação de saúde, no qual se trabalhou com:

1. A justificativa da priorização da situação de vulnerabilidade social;

2. A compreensão do processo histórico e social singular do território;

3. A definição dos objetivos dos atores envolvidos em relação à problemática em saúde desejos, limites, possibilidades;

4. 0 estabelecimento das ações que seriam efetivas para alcançar os objetivos propostos; e

5. A identificação de outros atores sociais e/ ou instituições importantes para o projeto.

Em seguida, partiu-se para a segunda etapa, planejamento/implementação, na qual se criou um espaço coletivo ampliado de gestão participativa e intersetorialidade para a construção compartilhada do PST, baseada na pactuação e corresponsabilização dos atores envolvidos para implementar o plano de ação, o que se deu por meio da realização do FSJ para uma cultura de paz, em 10 e 11 de dezembro de 2015, respectivamente na Escola Estadual "Monsenhor José Gerardo" e na Escola Municipal "Senador Carlos Jereissati", localizadas no Complexo Sinhá Sabóia.

A terceira etapa do PST, avaliação, possibilitou uma reflexão sobre o processo de implementação e os resultados alcançados em relação aos objetivos pactuados no planejamento.

\section{RESULTADOS E DISCUSSÃO}

Os resultados são organizados em 3 tópicos, com descrição das etapas realizadas no PST como vivenciado pelas autoras deste estudo enquanto membros da equipe de RMSF:

A. Preparando o PST: identificação e entendimento da situação de saúde;

B. Planejando e implementando o PST: o FSJ; e

C. Avaliando o PST: reflexão sobre a intervenção. 


\section{Preparando o PST: identificação e entendimento da situação de saúde}

A execução do PST partiu da identificação de uma situação-problema e de seus agravantes à saúde da população no território do Complexo Sinhá Sabóia, em Sobral, os quais se mostraram determinantes sociais em saúde.

Considera-se que os fatores de ordem social, econômica e comportamental, em geral, são determinantes sociais de saúde que podem influenciar positiva ou negativamente o processo saúde-doença dos indivíduos. Vale ressaltar que alguns deles podem decorrer de processos econômicos e decisões políticas, como no caso dos fatores físico-materiais, já que as disparidades socioeconômicas influenciam a saúde - a exemplo da escassez de recursos combinada a precário investimento em infraestrutura ${ }^{8}$.

Dentre os fatores supracitados se destaca a violência, que se manifesta de diferentes formas e gera forte impacto nas relações interpessoais, um fenômeno histórico na sociedade brasileira.

0 território do Complexo Sinhá Sabóia apresenta vulnerabilidades que expõem os adolescentes e jovens ao desemprego, ao uso de entorpecentes e à criminalização. A situação de violência preocupa a comunidade: os dados da territorialização relativos ao ano de 2016 indicam que $28 \%$ das mortes no bairro foram ocasionadas por causas externas - fato o que tem não só enlutado as famílias, mas deixado uma grande quantidade de indivíduos com sequelas limitantes ${ }^{9}$.

Com esse diagnóstico situacional, a violência se mostrou um desafio para a equipe de saúde, que organizou estratégias de gestão que visaram à integração de diversos setores no território. Como ferramenta de educação permanente e sob a perspectiva da clínica ampliada, os profissionais da ESF, junto com a RMSF, o Nasf-AB e o Conselho Local de Saúde do Complexo Sinhá Sabóia, utilizaram o PST como ferramenta para discutir a violência urbana e a promoção da cultura de paz.

A compreensão do processo histórico e socioeconômico da comunidade foi de suma importância na elaboração do PST, pois consolidou um entendimento aprofundado da situação/ necessidade de saúde na qual se almeja intervir, identificando o fenômeno que levou a determinada vulnerabilidade/risco ${ }^{1}$. Reflete-se que as situações de violência apresentam particularidades tanto quanto à localização demográfica como em relação

\section{...as disparidades socioeconômicas influenciam a saúde...}

às potencialidades para a promoção da paz.

A preparação dessa proposta envolveu articulação intersetorial, sobretudo entre as políticas de educação e saúde, que foram além das práticas multissetoriais, onde os vários setores se dedicam à resolução de um problema, mas não se observa a reciprocidade, partindo do entendimento de que a intersetorialidade implica coparticipação efetiva, ou seja, tomar parte na gestão, na produção e no usufruto compartilhado do projeto ${ }^{10}$.

As equipes da ESF e as equipes multiprofissionais (Nasf-AB e RMSF), as duas escolas municipais (Escola Paulo Aragão e Escola Senador Carlos Jereissati), a escola estadual (Escola Monsenhor José Gerardo) e a comunidade católica (Maranatá), reunidas enquanto Conselho Local de Saúde, foram os atores sociais envolvidos na construção do projeto, que teve como propósito a promoção da cultura de paz, por meio de participação popular e cogestão.

Destaca-se nesse processo a atuação da ESF como ator social, pois provoca, de fato e de direito, um relevante movimento de reorientação do modelo de atenção à saúde nas comunidades. Ao Nasf$A B$, que oferece apoio às equipes de ESF, compete o desenvolvimento de ações interdisciplinares e intersetoriais, educação permanente em saúde (dos profissionais e da população), atividades educativas no território, integralidade na assistência, participação social, educação popular, promoção da saúde e humanização ${ }^{11}$.

Já a RMSF atua na promoção da saúde junto à ESF e assume o papel de dispositivo de educação permanente e reorientação das lógicas técnicoassistenciais, potencializando as mudanças almejadas pelos profissionais da saúde, a fim de consolidar os princípios do Sistema Único de Saúde (SUS) ${ }^{12}$.

0 setor educação, o movimento estudantil e o setor religião, representados pelas escolas das redes municipal e estadual e pela comunidade católica Maranatá, tiveram grande parcela de contribuição no 
projeto, pois se engajaram nas discussões, sediando os debates e propondo resoluções para a temática cultura de paz, principalmente sob a perspectiva do protagonismo juvenil.

Como espaço de culminância do encontro dos diversos atores envolvidos no projeto, ressalta-se a contribuição do Conselho Local de Saúde, o espaço de participação mais próximo da comunidade, ou seja, o primeiro lugar em que as necessidades dos usuários dos serviços são identificadas e discutidas. Conselhos locais têm a possibilidade de dar voz às minorias e às pluralidades de seus interesses, inovando institucionalmente por meio de propostas de solução de problemas e mediação de conflitos locais.

Os conselhos locais de saúde vão além do modelo hegemônico de acompanhamento e fiscalização do sistema de saúde, constituindo espaços de interação e negociação entre os diversos atores no território, que deixam suas divergências e diferenças de lado para buscar um objetivo em comum: definir os problemas sociais e formular conjuntamente um plano de superação $0^{13}$.

Assim, tais conselhos fazem parte de uma estratégia de descentralização do Conselho Municipal de Saúde, para efetivar sua presença nos bairros ou na área de abrangência dos CSF e possibilitar maior participação dos usuários e profissionais dos serviços de saúde. Trata-se de espaços fundamentais para a democratização dos serviços nas unidades de saúde, promovendo o exercício da cidadania e o controle social em saúde.

$\mathrm{Na}$ etapa de preparação do PST se discutiu a situação de saúde no território, além de identificar, mobilizar, articular e congregar esforços para o desenvolvimento desse projeto. Nos primeiros encontros foi possivel conhecer a realidade e a necessidade de cada ator envolvido, por meio de oficinas iniciais de sensibilização com os profissionais para torná-los cientes dessa realidade.

\section{Conselhos locais têm a} possibilidade de dar voz às minorias e às pluralidades de seus interesses...
Foram realizadas duas oficinas iniciais: a primeira como reunião do Conselho Local de Saúde, onde se adotou como metodologia a árvore dos sonhos; e a segunda como ação de sensibilização das equipes do CSF Sinhá Sabóia, onde se recorreu ao teatro como recurso de problematização/sensibilização. A fase de preparação culminou na ideia de organizar um espaço para discutir essa questão, o que levou ao I Fórum Social da Juventude para uma Cultura de Paz.

\section{Planejando e implementando o PST: o FSJ}

Durante o planejamento e a implementação do PST, a participação dos atores sociais supracitados, reunidos enquanto Conselho Local de Saúde, mostrou-se primordial para a execução da proposta. As reuniões do Conselho Local de Saúde constituíram espaços de discussão para elencar as necessidades de saúde e as estratégias e os objetivos para sua abordagem. Nesse sentido, pôde-se estabelecer redes de cogestão e corresponsabilidade, instaurando um processo de cooperação e parceria entre os diversos atores sociais no território, culminando na organização e realização, no Complexo Sinhá Sabóia, do FSJ voltado a uma cultura de paz.

0 plano de ação elaborado durante a construção do PST tomou forma nesse fórum. 0 evento mobilizou diversos atores, que se corresponsabilizaram e assumiram o compromisso de participar ativamente na execução do plano. Em termos estratégicos, criaram-se comissões nas quais os atores puderam alocar-se (infraestrutura e logística, mobilização social, mobilização cultural, patrocínios, dentre outras), a fim de agilizar o processo e aprimorar o direcionamento do FSJ.

Esse evento ocorreu em 10 e 11 de dezembro de 2015 e contou com a participação de lideranças juvenis do bairro, profissionais da saúde, profissionais das escolas do território, grêmio estudantil, autoridades locais e membros da comunidade.

0 primeiro dia se dividiu em eixos temáticos com perguntas norteadoras sobre saúde, educação e lazer, dentre outros assuntos de relevância para a qualidade de vida da população. Nesse momento, os participantes se organizaram em grupos por eixos temáticos, para discutir propostas de melhoria para cada segmento. Cada grupo elaborou uma carta de propostas, posteriormente apresentada a todos em plenária geral.

Todas as propostas foram sintetizadas em uma carta geral do FSJ, apresentada no segundo dia 
do evento aos segmentos da gestão municipal convidados.

Várias propostas foram preparadas e alguns questionamentos da população foram esclarecidos pelas autoridades presentes. A carta do FSJ chegou ao conhecimento de outras autoridades que não estavam presentes no evento e foi apresentada no seminário de territorialização do município.

Discutiu-se que, na política de saúde, o planejamento assume uma tripla missão:

A. Ajudar os profissionais a refletir sobre suas práticas;

B. Construir cenários de curto, médio e longo alcance para as práticas e os resultados desejáveis no setor saúde; e

C. Organizar o processo de trabalho a fim de garantir a qualidade do que deve ser feito, viabilizando a transformação dessa realidade ${ }^{14}$.

0 FSJ levou os participantes a seguir engajados em discussões sobre a cultura de paz e estimulou a busca de alternativas para solucionar os diversos problemas que afligem o bairro, sob a perspectiva da promoção da paz e do ativismo juvenil. Dentre as propostas discutidas e aprovadas pelo evento, pode-se destacar a realização de oficinais sobre cultura de paz com crianças e adolescentes nas escolas e a intensificação das reivindicações junto às autoridades municipais no que concerne à criação e ao fortalecimento dos espaços de lazer e de cultura no território.

\section{Avaliando o PST: reflexão sobre a intervenção}

0 processo de avaliação se deu durante a construção do projeto, por meio de reflexão acerca das decisões tomadas junto com os atores envolvidos em todo o processo de identificação, planejamento e implementação. Além das avaliações realizadas no desenvolvimento do projeto, ao final de todas as ações houve avaliação geral, para apreciar toda a intervenção, permitindo que os diversos atores envolvidos se posicionassem e construíssem um parecer que se transformasse em ação.

Esse tipo de avaliação, contando com a participação de todos os atores envolvidos, mostrou-se uma ferramenta crucial para consolidar a cogestão, pois se contrapõe aos modelos de avaliação tradicional. Como evidencia a literatura pertinente ${ }^{15}$, há razoável consenso de que a abordagem tradicional se mostra ineficaz para produzir transformações sociais, assim,

\section{... a abordagem \\ tradicional se mostra \\ ineficaz para produzir \\ transformações \\ sociais,...}

espera-se que a abordagem participativa processe um tipo de aprendizado social que crie condições para a mudança.

\section{CONCLUSÃO}

A execução de um PST abrange uma série de elementos em diversos níveis de atuação em saúde. Pode-se afirmar tal ferramenta de planejamento em território específico é de grande valia para:

1. Identificar agravos, em diferentes contextos, que impactam a saúde da população;

2. Ampliar as redes intersetoriais de cuidado em saúde disponíveis no território;

3. Corresponsabilizar a população na solução de problemas de saúde considerados simples; e

4. Estimular as práticas de planejamento e avaliação das atividades propostas para melhoria da qualidade de vida da população.

0 PST desenvolvido no Complexo Sinhá Sabóia, por meio do FSJ voltado a uma cultura de paz, possibilitou a identificação de fragilidades locais e suas potencialidades, além de fortalecer as lideranças comunitárias, sobretudo as juvenis. Despertouse interesse em questões políticas necessárias para obter avanços em termos de saúde, educação, cultura e lazer - não apenas para a comunidade local, mas para outras esferas sociais. Isso possibilitou a congregação de diversos segmentos ligados à educação, à cultura, à saúde e ao poder público como um todo, estabelecendo diálogo sobre questões inerentes aos modos e às condições de vida da população.

Diante das inúmeras contribuições que 0 FSJ pode proporcionar para uma cultura de paz, percebe-se que o evento materializa o que pode ser produzido a partir de planejamento estratégico, envolvendo vários atores envolvidos na atenção à saúde: escolas, lideranças comunitárias, CSF, comunidades religiosas, grupos culturais, dentre 
outros. Tal iniciativa colabora para a ampliação de ações voltadas à cultura de paz no território de modo planejado e avalia as práticas adotadas para a melhoria da qualidade de vida da população.

\section{CONTRIBUIÇÃO DAS AUTORAS}

Todas as autoras contribuíram com a realização da pesquisa, o delineamento do estudo e a redação e revisão crítica do manuscrito.

\section{AGRADECIMENTOS}

As autoras agradecem a Jeferson Matos Floriano de Oliveira, Oseias Pereira Soares e Paulo Cesar de Moura Luz pelo apoio no desenvolvimento do estudo.

\section{REFERÊNCIAS}

1. Brasil. Diretrizes do NASF: núcleo de apoio à saúde da família. Brasília (DF): Ministério da Saúde; 2009. (Cadernos de Atenção Básica n. 27).

2. Verdi MIM, Freitas TG, Souza TT. Projeto de saúde no território. Florianópolis: Universidade Federal de Santa Catarina; 2012.

3. Trancredi FB, Barrios SRL, Ferreira JHG. Planejamento em saúde. São Paulo: Universidade de São Paulo; 1998. v. 2.

4. Mehry EE. Razão e planejamento. São Paulo: Hucitec; 1994.

5. Paim JS. Planejamento em saúde para não especialistas. Tratado de saúde coletiva. 2. ed. São Paulo: Hucitec; 2014.

6. Brasil. Avaliação na atenção básica em saúde: caminhos da institucionalização. Brasília (DF): Ministério da Saúde; 2005.

7. Serapioni M, Lopes CMN, Silva MGC. Avaliação em saúde. Epidemiologia e saúde. [place unknown]: Rouquayrol; 2012.

8. Buss PM, Pellegrini Filho A. A saúde e seus determinantes sociais. Physis (Rio J) [serial on the internet]. 2007 [cited 2018 Dec 11];17(1):77-93. Available from: http://www.scielo.br/pdf/physis/ v17n1/v17n1a06.pdf

9. Sobral (Município). Territorialização 2016. Sobral (CE): Secretaria da Saúde; 2016.

10. Dias MSA, Parente JRF, Vasconcelos MIO, Dias FAC. Intersetorialidade e Estratégia Saúde da Família: tudo ou quase nada a ver? Ciênc Saúde Colet [serial on the internet]. 2014 [cited 2018 Dec 11];19(11):437182. Available from: http://www.scielo.br/pdf/csc/ v19n11/1413-8123-csc-19-11-4371.pdf

11. Brasil. Política Nacional de Atenção Básica. Brasília (DF): Ministério da Saúde; 2012.

12. Silva CT, Terra MG, Kruse MHL, Camponogara $S$, Xavier MS. Residência multiprofissional como espaço intercessor para a educação permanente em saúde. Texto \& Contexto Enferm [serial on the internet]. 2016 [cited 2018 Dec 11];25(1):e2760014. Available from: http://www.scielo.br/pdf/tce/v25n1/01040707-tce-25-01-2760014.pdf

13. Ponte HMS, Lopes Filho J0, Feijão JRP, Souza $\mathrm{FL}$, Santos EV, Soares CHA. "O caminho se faz ao caminhar": processo de reativação de conselhos locais de saúde em Sobral, a partir do protagonismo cidadão. Saúde Soc [serial on the internet]. 2012 [cited 2018 Dec 11];21(Suppl 1):234-43. Available from: http://www.scielo.br/pdf/sausoc/v21s1/20. $\underline{p d f}$

14. Parente JRF. Planejamento participativo em saúde. Sanare (Sobral, Online) [serial on the internet]. 2011 [cited 2018 Dec 11];10(1):54-61. Available from: https://sanare.emnuvens.com.br/ sanare/article/view/144/136

15. Bursztyn I, Ribeiro JM. Avaliação participativa em programas de saúde: um modelo para o Programa de Saúde do Adolescente. Cad Saúde Pública [serial on the internet]. 2005 [cited 2018 Dec 11];21(2):40416. Available from: http://www.scielo.br/pdf/csp/ v21n2/07.pdf
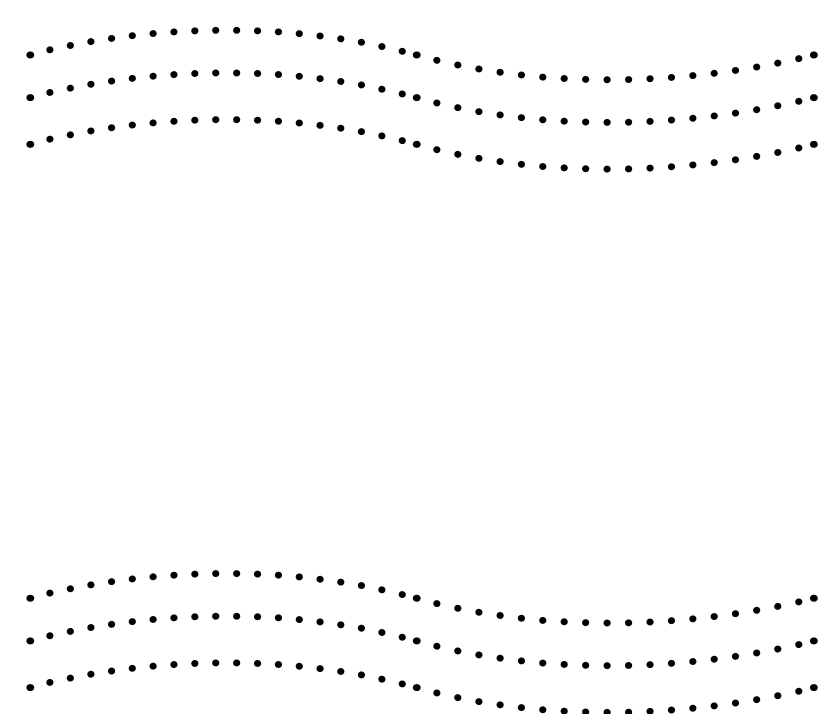